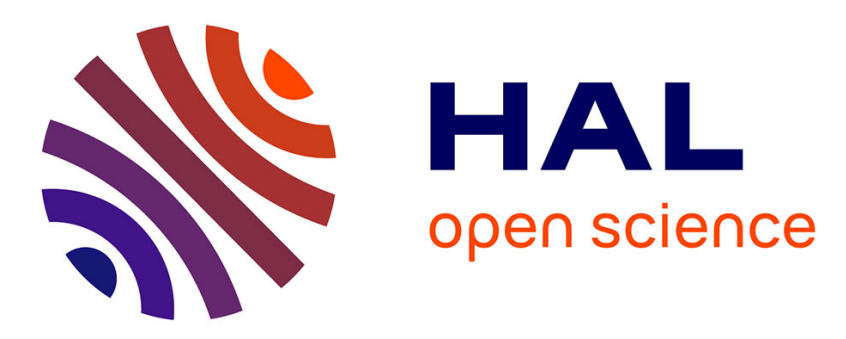

\title{
Identification and quantification of amino acids and related compounds based on Differential Mobility Spectrometry
}

Francis Berthias, Yali Wang, Eskander Alhajji, Bernard Rieul, Fathi Moussa, Jean-François Benoist, Philippe Maître

\section{To cite this version:}

Francis Berthias, Yali Wang, Eskander Alhajji, Bernard Rieul, Fathi Moussa, et al.. Identification and quantification of amino acids and related compounds based on Differential Mobility Spectrometry. Analyst, 2020, 145 (14), pp.4889-4900. 10.1039/d0an00377h . hal-02902596

\section{HAL Id: hal-02902596 https://hal.science/hal-02902596}

Submitted on 30 Dec 2020

HAL is a multi-disciplinary open access archive for the deposit and dissemination of scientific research documents, whether they are published or not. The documents may come from teaching and research institutions in France or abroad, or from public or private research centers.
L'archive ouverte pluridisciplinaire HAL, est destinée au dépôt et à la diffusion de documents scientifiques de niveau recherche, publiés ou non, émanant des établissements d'enseignement et de recherche français ou étrangers, des laboratoires publics ou privés. 


\section{Identification and quantification of amino acids and related compounds based on Differential Mobility Spectrometry}

Received 00th January 20xx, Accepted 00th January 20xx DOI: $10.1039 / \times 0 \times x 00000 x$

\author{
Francis Berthias, ${ }^{a}$ Yali Wang, ${ }^{a}$ Eskander Alhajji, ${ }^{a}$ Bernard Rieul, ${ }^{a}$ Fathi Moussa, ${ }^{a}$ Jean-François \\ Benoist, $^{\text {b,c }}$ and Philippe Maître*a
}

\section{Introduction}

Metabolomics-based strategies are routinely used for modern clinical research, allowing for more adequate diagnosis and prognosis. ${ }^{1,2}$ Quantitative and robust methods ${ }^{3}$ are required for the analysis of biomarkers of metabolic diseases (MDs) involved in a complex network of metabolic pathways. Amino acids (AAs) and related compounds constitute a class of biomarkers which is routinely analyzed for early diagnosis of MDs. ${ }^{4}$ Separation of these metabolites within, for example, urine or blood samples, has been initially performed by high-performance liquid chromatography (HPLC), which was coupled to spectrophotometric detection. ${ }^{5}$ For this detection purpose, postcolumn and precolumn derivatization had to be carried out, leading to time-consuming methods. Nowadays, metabolomics profiling is based on chromatographic separation coupled with tandem mass spectrometry (MS/MS). ${ }^{6}$ Nuclear magnetic

a. Université Paris-Saclay, CNRS, Institut de Chimie Physique, 91405, Orsay, France.

b. Université Paris-Saclay, Lipides, Systèmes Analytiques et Biologiques, 92296,

Châtenay-Malabry, France.

c. Service de biochimie hormonologie, Hôpital Universitaire Robert Debré, APHP, 48 Bd Serrurier, 75019 Paris.

Electronic Supplementary Information (ESI) available: composition of standard mixture; concentrations of AAs and related compounds in plasmas; composition and concentrations of calibration solutions; compensation voltage and collision cross section values for a set of AAs and related compounds; Limit of detection values; Day-to-day confidence interval on CV values. See DOI: 10.1039/x0xx00000x resonance (NMR) is also an important tool, but the main advantage of $\mathrm{MS} / \mathrm{MS}$ is its sensitivity that requires a minimal amount of biological material. ${ }^{7}$ Identification of the metabolites at their naturally occurring physiological concentrations is routinely achieved by comparing their fragmentation spectra with standard reference spectra. ${ }^{7}$ Some major drawbacks of the current methods, such as time-consuming procedures, poor reproducibility of the derivatization, and problems of retention have been shown to be overcome using hydrophilic interaction liquid chromatography (HILIC) for the separation. Rapid quantification of underivatized AAs in plasma using a HILIC column, ${ }^{8}$ and more recently mixed mode stationary phase and a binary gradient of elution, ${ }^{9}$ coupled with tandem massspectrometry have recently been reported. Despite these improvements providing high degree of selectivity, separation and identification of isomeric metabolites remains a difficult task. ${ }^{2}$ Additional or alternative dimensions of separation could thus be of interest for the separation of isobaric and isomeric metabolites. ${ }^{10-13}$

Ion mobility spectrometry (IMS) is an interesting alternative for the separation of small molecular weight ions such as ionized metabolites. ${ }^{14}$ Both time- and space-type IMS separation such as drift-time IMS (DT-IMS) and differential mobility spectrometry (DMS, also often referred to as Field Asymmetric Ion Mobility Spectrometry (FAIMS)), ${ }^{15}$ respectively, have been successfully used for the separation of metabolites. DT-IMS 
coupled to high resolution time of flight mass spectrometry has been employed for the profiling of human blood metabolome, and separation of 300 isomeric metabolites within various classes of metabolites could be achieved. ${ }^{16}$ Time separation of IMS (millisecond) allows for the coupling of IMS-MS/MS to chromatographic separation. An LC-IMS-MS/MS protocol has been recently proposed for metabolomics and lipidomics. ${ }^{17}$

Efforts toward separation of isobaric and isomeric species led to the development of high resolution instruments and/or methodologies. Within a decade, important progresses have been made with new types of instruments time- ${ }^{18,19}$ and spacemobility 20,21 separation. Significant enhancement of the resolving power can also be achieved by introducing a fraction of $\mathrm{He}^{22}$ or polar molecules ${ }^{23}$ in the carrier gas, as found in particular for AAs. ${ }^{24,} 25$ Separation of diastereoisomeric chiral compounds such as AAs and peptides has been addressed recently. For the separation of D/L AAs, both derivatization ${ }^{26}$ and formation of a proton bound dimers ${ }^{27}$ with a chiral agent have been shown to be successful. Mobility separation of peptides with 4-29 residues including a single D-AA at different residues and locations has recently been reported. ${ }^{28} \mathrm{High}$ resolution DMS has also been recently successfully employed for the separation of isobaric peptides containing one or two methylated arginine residues. ${ }^{29}$

Both space- and time-separation IMS approaches allow for a reduction of the signal-to-noise ratio. In addition, it has been shown that the number of false positive assignments can be reduced using DMS. ${ }^{30}$ DMS has also been shown to be more orthogonal to MS/MS than DT-IMS or related time-separation methods, in the case of peptides, for example. ${ }^{20,}{ }^{31}$ Coupled to $\mathrm{MS} / \mathrm{MS}$, it was shown that the coverage achieved with the DMS is comparable to that achieved with the chromatographic methods. ${ }^{32}$ DMS hyphenated to MS/MS has thus been used for analysis of complex biological fluids such as urine and plasma, and especially for the separation of co-eluting isomers. ${ }^{10,24}$ DMS-MS/MS workflows for lipid analysis has also been shown to overcome some limitation of LC-MS/MS such as low chromatographic resolution and the convolution of mass spectra from isomeric and isobaric species. ${ }^{12}$ Nevertheless, "despite several success stories concerning DMS in metabolomics, the application range of this technique is not fully understood. Systematic studies investigating the metabolome selectivity of DMS are required". ${ }^{32}$

Our aim is to evaluate the analytical performance of DMS hyphenated to MS/MS for the analysis of AAs and related compounds. More precisely, we will focus on the capacity of DMS for providing an additional metabolite identifier. As recently discussed by Barran and coworkers, ${ }^{33}$ this idea has been pursued using DT-IMS approaches, and it had been shown that mobility drift-time, ${ }^{34}$ or even collisional cross sections (CCS), ${ }^{35,} 36$ could be used for identification of pesticides. This idea of using CCS as identifier was further pursued for metabolites. $^{37-40}$ Using DMS, the additional identification parameter would be the so-called compensation voltage (CV) which has to be tuned for the transmission of a specific metabolite ion.
We first report on the analysis of the 20 common AAs, and the performance in terms of peak-to-peak resolution and peak capacity is compared to those using drift tube type IMS instruments, ${ }^{13-18}$ in particular for the separation of isobars and isomers. Potential effects of matrix or concentration of the analytes on the $\mathrm{CV}$ values are also evaluated, and found to be negligible. An interesting probe of the robustness of the method was also provided by the stability of the $\mathrm{CV}$ position for series of DMS-MS experiments carried over 6 months, during which the DMS device was often removed and replaced.

Isotopically labelled internal standards have been used for the quantification $^{41}$ of a selected sets of metabolites in human plasmas. The method linearity, intra-assay and inter-assay precision, detection limit, quantification limit and trueness analysis were found to be adequate in both physiological and pathological conditions. Concentration levels of metabolites derived with our DMS-MS protocols were found to be in good agreement with those obtained with routine LC-MS/MS protocols used for the diagnosis of MDs at the Hospital Robert Debré (Paris).

\section{Experimental methods}

Reagents. Methanol and water were used as solvent (50:50, vol:vol) and acidified with formic acid (0.5\%). Methanol was purchased from Merck KGaA (Darmstadt, Germany) and formic acid from Fisher Scientific (Hampton, New Hampshire, USA), both are reagent grade. Deionized water is obtained using Millipore Direct-Q 3 UV (Lilipore corporation, Burlington, Massachusetts, USA). Certified graduated pipettes were used throughout for the sample preparation.

Mixture of reference standards. A standard mixture made of 33 AAs and related compounds as listed in Table S1 (left column) was used for calibration. This mixture is based on a commercial AA standard solution (Type B, AN-II purchased from FUJIFILM Wako Pure Chemical Industries, Osaka, Japan). It was complemented by glutamine (Gln), asparagine (Asn), tryptophan (Trp), pipecolic acid, alloisoleucine (Ail), and sulfocysteine which were purchased from Sigma-Aldrich (St. Louis, Missouri, USA). Calibration curves were obtained using 6 calibration concentration levels in plasma.

Mixture of Internal standards. A mixture of stable isotope internal standards (IS) was made using reference compounds from Eurisitop (Saint Aubin, France). The list of these IS as well as their final concentrations in the samples is given in Table S1 (right column). This IS mixture was used for the calibration and quantification protocols.

Samples and subjects. Blood samples were obtained from patients suspected of MD based on routine diagnostic. Blood was collected by venous puncture into heparin containing tubes. In practice, blood was centrifuged rapidly after collection. Plasma was subsequently deproteinized by adding sulfosalicylic acid $30 \%(10: 1 \mathrm{v}: \mathrm{v})$ and then stored at $-20^{\circ} \mathrm{C}$. Just before analysis, samples were diluted in acidified water:methanol (50:50 v:v) solution, and 20-fold diluted plasma samples were used for electrospray injection. 
Four plasma samples (P1, P2, P3, and P4) not specifically collected for validation of this study were analyzed anonymously. The quality control P1 plasma is a pool of plasmas taken from healthy volunteers. Plasma P2 and P3 were collected from patients with Phenylketonuria and Tyrosinemia, respectively. $\mathrm{P} 4$ is a random plasma.

Plasma samples were collected for diagnosis purpose as part of the patient's routine care. Informed consent was obtained from patients for the use of leftover samples for analytical developments. The study was approved by the ethics committee of Robert Debré University Hospital (Paris, France), and in compliance with French Public health regulations (Code de la Santé Publique - Article L1121-3, amended by Law n²0112012, December 29, 2011 - Article 5).

Concentration of the AAs of interest in these plasmas has been quantified by a liquid chromatography tandem mass spectrometry (LC-MS/MS) method validated according to the ISO 15189 standards. These quantification experiments were performed in an accredited university hospital laboratory where MD diagnoses are routinely performed. In brief, after butylation, compounds are separated by reverse phase liquid chromatography, then ionized by electrospray in the positive mode and identified by multiple reaction monitoring (MRM). Concentrations in samples and corresponding uncertainties for the molecules are given in Table S2.

Differential Mobility Spectrometry (DMS) coupled to MS/MS instrument. DMS-MS experiments were carried out using a modified Esquire 3000+ quadrupole ion trap mass spectrometer (Bruker-Daltonics, Bremen, Germany) featuring an ElectroSpray Ionization (ESI) source. ${ }^{42}$ The setup is based on the one developed by Glish and coworkers. ${ }^{43}$ Our home-built DMS device has been successfully used for the separation and identification of isomers of alanine ${ }^{44}$ and aminobutyric acids. ${ }^{45}$ The DMS housing is mounted between the ESI emitter and the glass transfer capillary of the mass spectrometer. It is composed of two parallel electrodes $(0.7 \times 6 \times 20 \mathrm{~mm}$ for gap height, width, and length, respectively) between which ions are transported from the atmospheric pressure to a $\sim 10^{-3} \mathrm{mbar}$ source region pressure of the mass spectrometer through the glass transfer capillary. In order to enhance the transmission of ions, the standard glass transfer capillary has been replaced with a custom flared glass transfer capillary. ${ }^{33,34}$ The DMS cell is designed such as to replace the spray shield. The $\mathrm{N}_{2}$ desolvation gas is redirected through the outer housing of the DMS device in such a way that part of the $\mathrm{N}_{2}$ flow $\left(\sim 0.9\right.$ I. $\left.\mathrm{min}^{-1}\right)$ is used to transport the ions between the electrodes towards the glass capillary. An NIGen LCMS 40-1 (Claind, Tremezzina, Italy) $\mathrm{N}_{2}$ generator was used to produce the $\mathrm{N}_{2}$ gas with a purity up to $99.99 \%$. Since ion mobility is very sensitive to the composition of the carrier gas, a custom built chamber has been built to accommodate the DMS device, and allowing to adjust the position of the ESI nebulizer needle with respect to the entrance of the DMS device.

Identical positive ESI conditions have been used throughout. The voltage of the ESI emitter was set to $4.5 \mathrm{kV}$ and the entrance glass transfer capillary was grounded. The flow rate of the sample was $40 \mu \mathrm{L} . \mathrm{h}^{-1}$.
DMS allows for space separation of the ions, and the working principles can be found, for example, in a textbook ${ }^{46}$ and a review. ${ }^{15}$ In practice, a bisinusoidal waveform is applied between the electrodes thus creating an alternating electrical field perpendicularly to the direction of the carrier gas flow. This asymmetric radiofrequency is generated by applying a sinusoidal waveform at $1.7 \mathrm{MHz}$ to one of the DMS electrodes. A second sinusoidal waveform at the second harmonic, phase shifted by approximately $90^{\circ}$, and at $50 \%$ of the amplitude of the first sinusoidal waveform is applied to the second electrode. The zero-to-peak voltage $\left(\mathrm{V}_{\mathrm{Op}}\right)$ is referred to dispersion voltage (DV). lons are transmitted through the DMS device only if the transverse displacements under low and high electric field offset one another. Transmission of ions of interest can be achieved by applying a DC offset to one DMS electrode, referred to as compensation voltage (CV). DMS-filtered ions are then transmitted to the ion trap where they are accumulated before being subjected to MS/MS sequences.

Considering the gap between the electrodes $(0.7 \mathrm{~mm})$, and assuming that the carrier gas is near atmospheric pressure and a temperature around $70^{\circ} \mathrm{C}$ (measured temperature of the electrodes using a thermal camera), the reduced electric field value corresponding to a voltage of $1800 \mathrm{~V}$ can be estimated to $\sim 120 \mathrm{Td}$. In the following, the DMS spectra correspond to extracted ion chromatogram plotted as a function of the CV value, where a $1 \mathrm{~V} \mathrm{CV}$ value corresponds to an electric field of $1.43 \mathrm{kV} . \mathrm{m}^{-1}$ or $\sim 0.07 \mathrm{Td}$

DMS Spectra: Data processing. DMS spectra are generated by plotting extracted ion chromatograms (XIC) as a function of CV value, which is scanned using a $0.1 \mathrm{~V}$ increment. DMS peak centroid (CV) and full width at half-maximum (FWHM) are extracted by fitting each DMS spectrum using Gaussian functions. The fitting procedure is performed using a homebuilt software based on SciPy libraries. An optimization algorithm based on a sequential least squares programming (SLSQP) method is used for the Gaussian fits.

DMS spectra of the components of the standard mixture and dispersion plots of individual AA. Unless otherwise stated, DMS spectra presented here were recorded with a DV value set to $1.8 \mathrm{kV}$. All the $\mathrm{CV}$ values corresponding to optimal transmission of a particular metabolite given in the text refer to these conditions.

Dispersion plots, i.e. evolution of $\mathrm{CV}$ value for the optimal transmission of each AA as a function of the DV value, were recorded. DMS spectra were recorded at different DV values ranging from 600 to $1800 \mathrm{kV}$. Solutions of AAs were prepared in methanol:water solvent mixture acidified with formic acid $(0.5 \%)$ at a final concentration of $100 \mu \mathrm{M}$.

Calibration curve and protocol for quantification of metabolites in Plasma. The set of standards of AAs and related compounds (Table S1) was spiked into the P1 plasma at six dilution factors spanning 2 orders of magnitude of concentration (Table S3). Except for pipecolic acid and alloisoleucine, which are found at very low concentrations in plasma, the dilution levels were chosen so that the pathophysiological AA concentrations in analyzed plasma lie in the range of the spiked $\mathrm{P} 1$ solutions. 
In practice, the calibration solutions spiked in the P1 plasma sample at a final $1 / 20$ dilution factor were prepared as follow. Following the deproteinization with sulfosalicylic acid, $13.8 \mu \mathrm{L}$ of the supernatant was mixed with $25 \mu \mathrm{L}$ of the IS mixture, and diluted in a water:methanol (50:50) solution containing standards and $1.25 \mu \mathrm{L}$ of formic acid to reach a total volume of $250 \mu \mathrm{L}$.

Linear calibration curve parameters were obtained from the plot of the AA/related IS peak area ratio versus the nominal AA concentration. The CV, FWHM, and DMS peak area values were derived from the above mentioned fitting procedure.

Quantification of AAs and related compounds in the four plasma samples (P1-P4) was performed. The sample preparation protocol is similar to that used for the calibration. $13.8 \mu \mathrm{L}$ of deproteinized plasma was added to $25 \mu \mathrm{L}$ of IS mixture and diluted in acidified water:methanol to a final volume of $250 \mu \mathrm{L}$. In order to check for potential carry-over, a blank run was systematically performed between two plasma runs. The concentrations of unknown samples were calculated from their corresponding area ratios using the corresponding calibration curve.

\section{Results and discussion}

The aim of this work is twofold. First, it is to evaluate whether the $\mathrm{CV}$ parameter could provide a means for the identification of metabolites. Our second goal is the absolute quantification of AAs and related compounds for targeted metabolomics analysis of plasma samples from healthy or MD patients. In this perspective, the performance of DMS for the separation of AAs is first evaluated, with a special focus on peak capacity and isobars/isomers resolution. Then, the compensation voltage value of the AAs and related compounds within a mixture of standards are determined, in both water:methanol solution and plasma. Based on the good robustness and repeatability of the
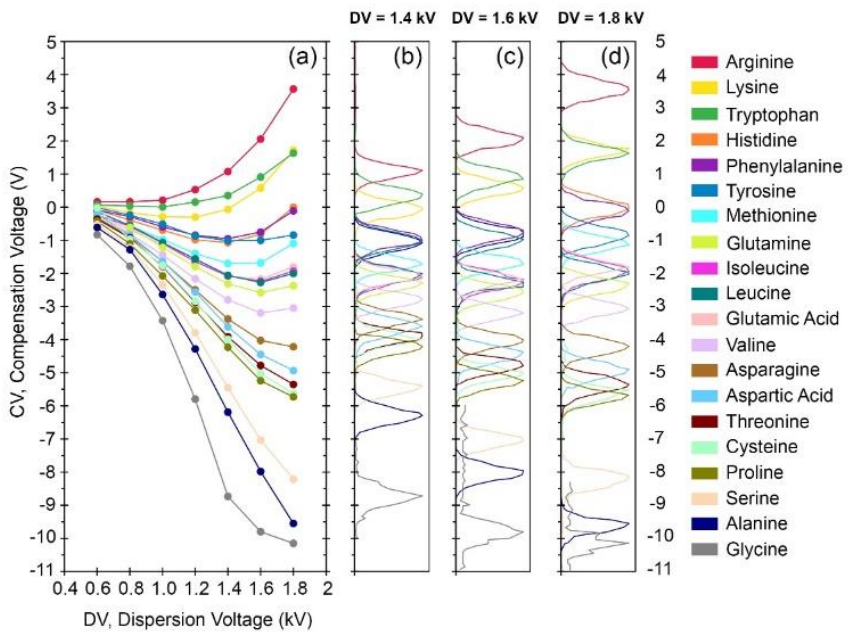

Fig. 1 Differential ion mobility data for the 20 protonated AAs recorded at different dispersion voltage (DV) using $\mathrm{N}_{2}$ as carrier gas. (a) DMS dispersion plots, or evolution of CV as a function of DV; DMS spectra for each individual $\mathrm{AA}$ at $\mathrm{DV}=1.4,1.6,1.8 \mathrm{kV}$ are given in panels (b), (c), and (d), respectively. A $\mathrm{CV}$ value of $1 \mathrm{~V}$ corresponds to an electric field of $1.43 \mathrm{kV} . \mathrm{m}^{-1}$ or $\sim 0.07 \mathrm{Td}$. To facilitate visualization, each DMS peak is individually normalized. measurements, the calibration results are then presented. Finally, the quantification of a set of AAs and related compounds relevant for the diagnosis of MDs is presented for four sets of plasma samples.

DMS separation of the $\mathbf{2 0}$ common AAs. In order to probe the effect of the DV value on peak capacity and resolution, DMS spectra were recorded at DV values ranging from $0.6 \mathrm{kV}$ to $1.8 \mathrm{kV}$. The set values of flow rate of the $\mathrm{N}_{2}$ desolvation gas and its temperature are $6.5 \mathrm{~L} . \mathrm{min}^{-1}$ and $220^{\circ} \mathrm{C}$, respectively. This was found to provide a good balance between DMS resolution and ion transmission. Results of separation are reported in Figure 1. DMS dispersion plots, i.e. the evolution of $\mathrm{CV}$ as a function of DV for each individual AA, are reported in Figure 1 . Three classes of AAs can be distinguished based on the evolution of CV as a function of DV (Figure 1a). These three types of behaviors have been observed for other systems ${ }^{47}$ and interpretations have been proposed ${ }^{15}, 46$ based on the interactions between ions with the carrier gas. These three types of ions, often referred to as A, B, or C ions, are observed for AAs under the present DMS conditions. ${ }^{47}$ Protonated asparagine, aspartic acid, threonine, cysteine, proline, serine, alanine and glycine are associated to type $A$ ions, for which ionmolecule clustering and declustering are supposed to occur at low and high electric field, respectively. Type $C$ refers to ions with a decreasing mobility coefficient $K$ as a function of the electric field strength. These ions are supposed to experience hard sphere type collisions with the carrier gas. Protonated arginine and tryptophan were found to be the only two systems of type $\mathrm{C}$ under our experimental conditions. For type $\mathrm{B}$, the ion mobility initially increases, reaches a maximum and decreases for larger DV values. As a result, the DMS dispersion curve presents a minimum. It has recently been proposed that the minimum CV values can be correlated to ion-solvent binding energy. ${ }^{48}$ Protonated lysine, histidine, phenylalanine, tyrosine, methionine, glutamine, isoleucine, leucine, glutamic acid and valine are type $B$ ions. Note that some of these ions have been already found to behave as B type ions, such as protonated leucine ${ }^{49}$ and protonated glutamic acid. ${ }^{50}$

DMS spectra of each individual AA for three DV values, 1.4, 1.6, and $1.8 \mathrm{kV}$, are given in Figures $1 \mathrm{~b}-\mathrm{d}$, respectively. The width (FWHM) of the DMS peaks does not vary with the DV value. A nearly linear increase $\left(R^{2}=0.88\right)$ of the FWHM value with $m / z$ is observed, the values for the AAs ranging from 0.52 (Gly) to 0.88 $\mathrm{V}(\operatorname{Trp})$.

A plot of the $\mathrm{CV}$ values of the set of AAs, and also some related compounds which can be found in plasmas, as a function of $\mathrm{m} / \mathrm{z}$ is given in Figure 2. Overall, the observed trend is quite similar to that observed for the collision cross section (CCS) values $\left(\AA^{2}\right)$ derived using time-separation IMS instrument. ${ }^{17,33,38,51}$ With increasing $m / z$, the $C V$ value increases from $A-$, to $B$-, and C-type protonated AAs ions.

Separation of AAs: DMS versus IMS peak capacity. Separation of protonated AAs has been investigated using space ${ }^{24,25}$ and time separation based ion mobility instrument such as drifttube $^{52,53}$ or radio frequency-confining drift cell, i.e. with a Traveling Wave Ion Mobility Spectrometer (TWIMS). ${ }^{51}$ In the 
latter study, the effect of the nature $\left(\mathrm{He}, \mathrm{Ar}, \mathrm{N}_{2}, \mathrm{CO}_{2}\right.$, and $\left.\mathrm{N}_{2} \mathrm{O}\right)$ of the drift gas on both peak-to-peak resolution $\left(R_{p p}\right)$ and peak capacity $\left(P_{c}\right)$ has been evaluated. $R_{p p}$ and $P_{c}$ are defined in equations (1) and (2), where $C V_{\min }$ and $w_{\min }\left(C V_{\max }\right.$ and $\left.w_{\max }\right)$ are the CV peak position and full width at half maximum (FWHM) values, respectively, corresponding to the minimum (maximum) $\mathrm{CV}$ value.

$$
\begin{array}{r}
R_{p p}=1.178 \times \frac{C V_{1}-C V_{2}}{w_{1}+w_{2}} \\
P_{C}=2 \times \frac{C V_{\max }-C V_{\min }}{w_{1}+w_{2}}
\end{array}
$$

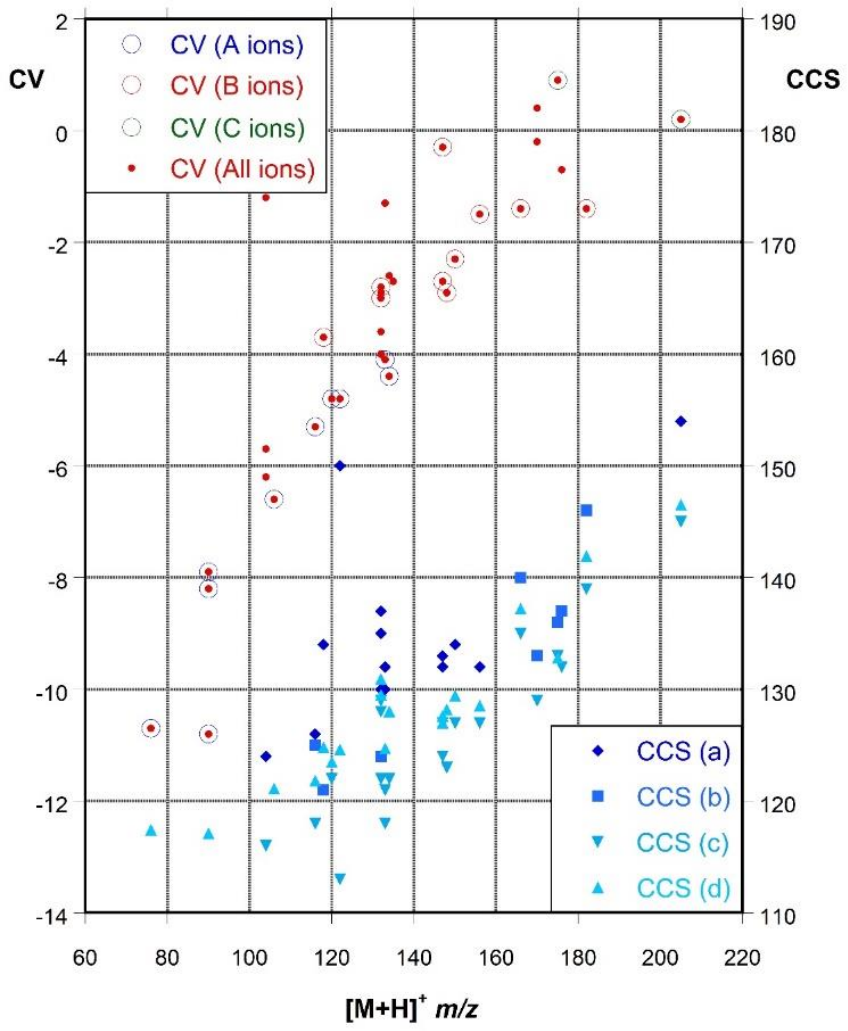

Fig. $2 \mathrm{CV}$ and CCS as a function of $\mathrm{m} / \mathrm{z}$ for a selected set of AAs and related species (See list in Table S4). CV values (in V), $1 \mathrm{~V}$ corresponding to an electric field of $1.43 \mathrm{kV} \cdot \mathrm{m}^{-1}$ or $\sim 0.07 \mathrm{Td}$. CCS (in $\AA^{2}$ ) are taken from (a) ref. 36, (b) ref. 31 , (c) ref. 17 , and (d) ref. 49.

As can be seen in Figure $1, P_{c}$ increases with $D V$, from $P_{c}=18.1$ at $1.4 \mathrm{kV}$ to $P_{c}=23.8$ at $1.8 \mathrm{kV}$. For more details, the evolution of $P_{c}$ (black curves) and $R_{p p}$ (blue curve) as a function of the DV value is shown in Figure $\mathrm{S} 1$. The best separation performance is observed for DV value of $1.8 \mathrm{kV}$ except for few AA pairs. For example, protonated lysine (B type) and protonated tryptophan (C type) are poorly separated at $1.8 \mathrm{kV}\left(R_{\mathrm{pp}}=0.1\right)$, while a better separation is observed at lower DV $\left(R_{p p}=0.48\right.$ at $\left.D V=1.2 \mathrm{kV}\right)$. In a consistent manner, the fraction of the pairwise combinations of protonated AAs which are baseline resolved $\left(R_{p p}=1.5\right)$ increases from $29 \%$ at $D V=1 \mathrm{kV}$ to $81 \%$ at $D V=1.8 \mathrm{~V}$. It can thus be concluded that these $R_{p p}$ and $P_{c}$ values are very close to those estimated $\left(\mathrm{P}_{\mathrm{c}} \sim 15\right.$ and $\sim 80 \%$ resolved $\mathrm{AAs}$ $\left.\left(R_{\mathrm{pp}}>1.0\right)\right)$ for a high performance drift tube instrument using $\mathrm{N}_{2}$ as a carrier gas. ${ }^{51}$
CV as metabolite identifier: no matrix, nor concentration effects. DMS data recorded for a selected set of AAs and related metabolites in two matrices, plasma and water:methanol, are reported in Figure 3. 2D plot of $m / z$ as a function of CV values for a mixture of standards diluted in water:methanol is given in Figure 3a. The corresponding 2D plot for the pool of plasma sample (P1) containing IS is given in panel $b$. In each case, the mass spectrum integrated over the whole $\mathrm{CV}$ range is given on the right hand side in panels d) and e), respectively. XIC for a selected set of $\mathrm{m} / \mathrm{z}$ values specific of molecules of interest for plasma study are given in Figure 3c. The blue and red lines correspond to standards in water:methanol solution and plasma sample, respectively. All the curves are normalized to maximum and smoothed using a rolling average over two points.

As expected, a higher density of contributions both in CV and $\mathrm{m} / \mathrm{z}$ dimensions is observed in plasma. Nethertheless, the 2D plots of standards and plasma show common features. As can be seen in Figure 3c, no significant matrix effect on CV position nor in the DMS peak width are observed. This is confirmed through the comparison of the CV values determined for a selected set of metabolites in a plasma or diluted in water:methanol solution (Table 1). The standard deviation (SD) derived using a set of 36 plasma measurements is smaller or equal to $0.1 \mathrm{~V}$, except for glycine $(0.2 \mathrm{~V})$, alanine $(0.3 \mathrm{~V})$, and serine $(0.2 \mathrm{~V})$. Considering the SD values, there is an excellent agreement between the two sets of values, and thus no evidence of matrix effects.

Concentration effects on DMS peak width and position could also be expected. DMS peaks were characterized for a set of AAs and related compounds when spiked in plasma P1 using six dilution factors spanning two orders of magnitude of concentration (1-100 $\mu \mathrm{mol})$. Overall, both FWHM and position of DMS peaks were found constant over these experiments. For the molecules of interest here, the mean value of $\mathrm{CV}$ standard deviation was $0.1 \mathrm{~V}$. The maximum $\mathrm{CV}$ standard deviation $(0.18 \mathrm{~V})$ was found for protonated glycine. Similarly, the peak width was also found not to be correlated with the concentration value. The mean FWHM value was $0.72 \mathrm{~V}$, and the standard deviation was $0.1 \mathrm{~V}$ in the concentration range examined.

A full assignment of the XIC is out of the scope of this study, and unassigned peaks are labeled with a "*" symbol in Figure 3c. DMS peaks associated to positive CV values, however, have to be analyzed carefully. It is indeed well-known that protonated homo- and hetero-dimers are typically transmitted at positive $\mathrm{CV}$ values. ${ }^{54}$ And these dimers are partially fragmented in the source region, at the exit of the glass capillary, just downstream of the DMS device. ${ }^{54}$ Due to this so-called "post-DMS fragmentation", their corresponding protonated monomers are observed at the corresponding transmission CV of the dimers. ${ }^{55}$ Hence, in addition to the DMS peak of a given protonated monomer at $m / z$, peaks are also observed at positive CV values corresponding to maximum transmission of their proton bound dimers. This is evidenced in Figures $3 a$ and $3 b$ where the $2 \mathrm{D}$ 


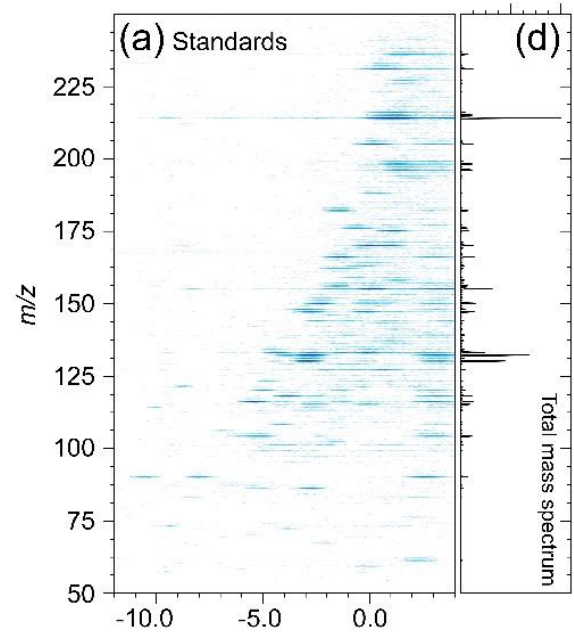

$\mathrm{CV}$, Compensation Voltage $(\mathrm{V})$

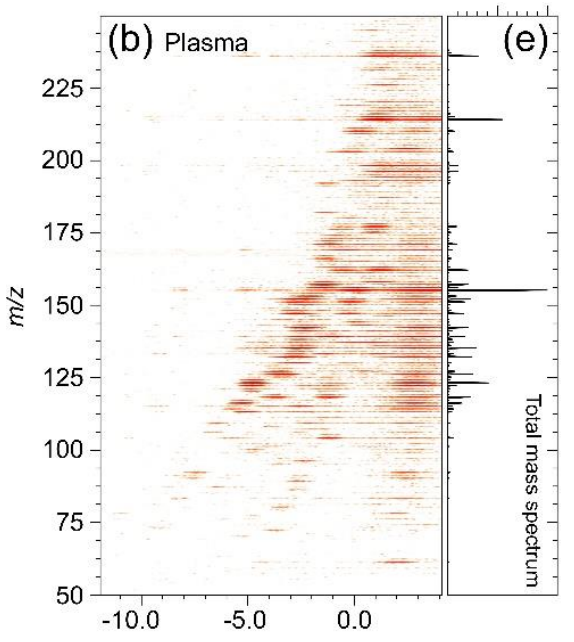

$\mathrm{CV}$, Compensation Voltage ( $\mathrm{V})$

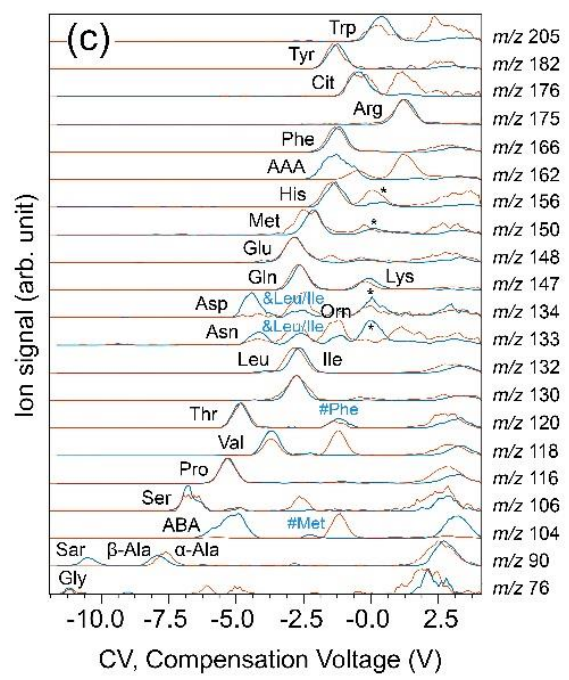

Fig. 3 DMS data recorded for a set of standards (AAs and related compounds) in water:methanol and for a plasma sample (P1) containing IS. The corresponding $2 \mathrm{D}$ plots, with $\mathrm{m} / \mathrm{z}$ as a function of $\mathrm{CV}$, are given in panels $\mathrm{a}$ ) and b), respectively. Extracted lon chromatograms (XIC) of a selected set of metabolites of interest are given in panel c) where data for the plasma (red) and standards in water:methanol solution (blue) are superimposed. Mass spectra integrated over the CV range corresponding to 2D plots of panels a) and b) are given in panels d) and e), respectively. Peak assignment in the XIC panel c) is provided. 3-letter nomenclature symbols are used for AAs, as well as for Aminoadipic acid (AAA), Aminobutyric acid (ABA), Citrulline (Cit), Ornithine (Orn), and Sarcosine (Sar). Peaks corresponding to post-DMS (in-source) fragments or also first and second isotopic peaks are labelled with an "\#" and an " $\&$ " symbol, respectively. Unassigned DMS peaks are symbolized by a "*" mark. A CV value of $1 \mathrm{~V}$ corresponds to an electric field of $1.43 \mathrm{kV} \cdot \mathrm{m}^{-1}$ or $~ 0.07 \mathrm{Td}$.

plots in the positive $\mathrm{CV}$ range do not significantly depend on the $m / z$ value.

In addition, a slight fragmentation of some protonated monomer of standards may also occur. This is the case for phenylalanine $(\mathrm{m} / \mathrm{z} 166 \rightarrow 120)$, and methionine $(\mathrm{m} / \mathrm{z} 150 \rightarrow 104)$ as shown in Figure 3c with "\#” marks. These fragments can be easily assigned because they are generated through post-DMS dissociation and are thus found at the $\mathrm{CV}$ position of the corresponding precursor ion. Finally, one must also pay attention to isotopic peaks (see " $\&$ " in Figure $3 c$ ). In the case of arginine $\left(\mathrm{MH}^{+} \mathrm{m} / \mathrm{z}\right.$ 175), which is abundant in the plasma and observed at $0.95 \mathrm{~V}$, a significant contribution associated with the first isotopic peak is also observed in the $\mathrm{m} / \mathrm{z} 176 \mathrm{XIC}$. Similarly, contributions of the first and second isotopic peaks of leucine/isoleucine are found in the $\mathrm{m} / \mathrm{z} 133$ and $134 \mathrm{XIC}$, respectively.

Separation of isobaric and isomeric AAs and related species. Considering the limited mass-resolution of our quadrupole ion trap instrument, the separation of isobaric species is an important issue. The performance of DMS relative to drift-tube instruments can be assessed by inspecting Figure 2 where $\mathrm{CV}$ and CCS are plotted as a function of $m / z$. The corresponding values for a selected set of isobaric and/or isomeric species discussed below are given in Table S4.

Apparent disagreements in terms of DMS peak position are in fact the result of the different relative concentrations of (partially) unresolved isomeric compounds in the two matrices (water:methanol and plasma). This is the case for example for the broad DMS peak observed near $-7.5 \vee$ for $m / z \quad 90$ ions (Figure 3c). It results from contribution of $\alpha$ - and $\beta$-alanine, ${ }^{44}$ the latter being observed at slightly more negative $(-0.3 \mathrm{~V}) \mathrm{CV}$ value than the former. The difference between the two $\mathrm{m} / \mathrm{z} 90$ $\mathrm{XIC}$ near $-7.5 \mathrm{~V}$ (Figure 3c) is thus simply due to the fact that $\beta$ a component of our standard mixture. More important is the issue of the separation of $\alpha$-alanine and sarcosine which cannot be distinguished based on their fragmentation mass spectra. These two ions were found to be baseline separated, the sarcosine peak being observed at $-10.6 \mathrm{~V}$. This DMS peak in observed for the standard mixture, but not for the plasma, where Sarcosine is expected at a very low concentration.

A similar issue occurs in the XIC at $\mathrm{m} / z 132$ (Figure 3c). Indeed, leucine/isoleucine have similar concentrations in the standard mixture, but not in the plasma. As a result, the shape of the unresolved DMS peak in the XIC at $m / z 132$ (Figure 3c) changes accordingly. In addition, note that Allo-isoleucine (Ail) is also included in the standard mixture, while it is not expected in plasma P1 which is from healthy volunteers. Measurements on each individual Leu isomer were performed, and the $\mathrm{CV}$ values of Leu, Ile, and Ail were found to be -3.0, -2.8, and $-2.9 \mathrm{~V}$, respectively. Better peak-to-peak resolution is obtained with isobaric cis- and trans-hydroxyproline, which were found to be transmitted at -3.6 and $-4.0 \mathrm{~V}$, respectively.

For $m / z$ 133, three baseline separated peaks were observed at metabolites, the peaks at -4.1 and $-1.3 \mathrm{~V}$ can be assigned to Asn and Orn, respectively. As can be seen in Figure 2, the DMS resolution is better than that obtained with a drift-tube instrument. For example, Orn and Asn measured CCS were found to be 130 and $132 \AA^{2}$, respectively. ${ }^{38}$

Similarly, protonated glutamine and lysine were found to be baseline resolved and found at -2.7 and $-0.3 \mathrm{~V}$, respectively. Their measured CCS, however, were found very close (133 and $132 \AA^{2}$, respectively). ${ }^{38} \mathrm{DMS}$ performance in terms of separation of isomeric species can be illustrated with 1- and 3- alanine is only found as trace amounts in the plasma while it is $-4.0,-2.6$, and $-1.4 \mathrm{~V}$. Based on measurements on individual 
methylhistidine. DMS measurement on individual isomers show that they are found at -0.2 and $0.4 \mathrm{~V}$.

CV as metabolite identifier: reproducibility and robustness. The robustness of the method in terms of accuracy on the peak position over time is also an important issue in the perspective of using CV as a metabolite identifier. Figure 4 illustrates the stability of DMS peak position for a selected set of six AAs over a period of 6 months. These DMS spectra include experiments performed with plasma (filled circles) and standards in water:methanol solutions (open circles). The mean CV and associated standard deviation values of the DMS peaks given in Figure 4 can be found in Table 1 . The maximum standard deviations were found for serine $(0.15 \mathrm{~V})$ and proline $(0.12 \mathrm{~V})$ while other standard deviation values are smaller than the $\mathrm{CV}$ scan step (0.1 V).

DMS specificity. The filtering effect of DMS over a short range of $\mathrm{m} / \mathrm{z}$ values containing protonated proline $(\mathrm{m} / \mathrm{z} 116)$ and valine $(\mathrm{m} / z \mathrm{118})$ is illustrated in Figure 5 . The mass spectrum of a pool of plasmas (P1 sample) recorded with RF off and $C V=0$ (DMS Off or transparent mode) is given in black in Figure 5a. A significant reduction of background signal is observed when DMS is turned on with the CV value set to -3.7 and $-5.3 \mathrm{~V}$ for optimal transmission of protonated valine (blue) and proline (red) as illustrated in Figure 5. The mass spectra in panel b) are those recorded at the optimal CV value, while mass spectra integrated over the targeted DMS peak are given in panel c).

Table 1. Compensation voltage (CV) values recorded for a selected set of metabolites in a plasma or diluted in water:methanol solution. Mean $\mathrm{CV}$ and the corresponding standard deviation (SD) are given in Volt unit. Day-to-day reproducibility of the DMS peaks and correspondence between CV and electric field are given in Table S6.

\begin{tabular}{|l|c|c|c|c|}
\hline & \multicolumn{2}{|c|}{ Plasma } & \multicolumn{2}{c|}{ Water:MeOH } \\
\hline & CV & SD & CV & SD \\
\hline glycine & -10.7 & 0.2 & & \\
\hline
\end{tabular}

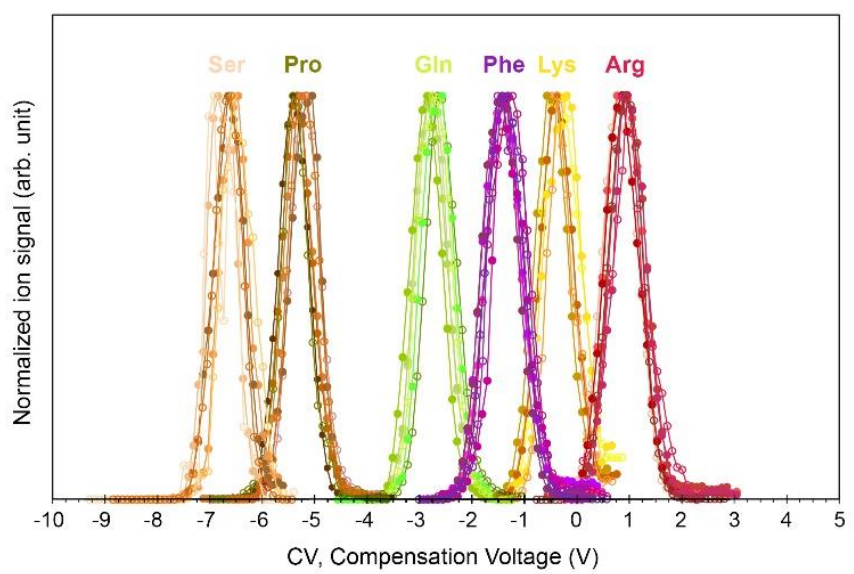

Fig. 4 Selected DMS spectra recorded for serine (Ser), proline (Pro), glutamine (Gln), phenylalanine (Phe), lysine (Lys) and arginine (Arg) over a period of 6 months. Experiments were performed with plasma samples (filled circles) and standards of AAs and related compounds in water:methanol solutions (open circles). The mean CV and associated standard deviation values are given in Table 1. A CV value of $1 \mathrm{~V}$ corresponds to an electric field of $1.43 \mathrm{kV} \cdot \mathrm{m}^{-1}$ or $\sim 0.07 \mathrm{Td}$.

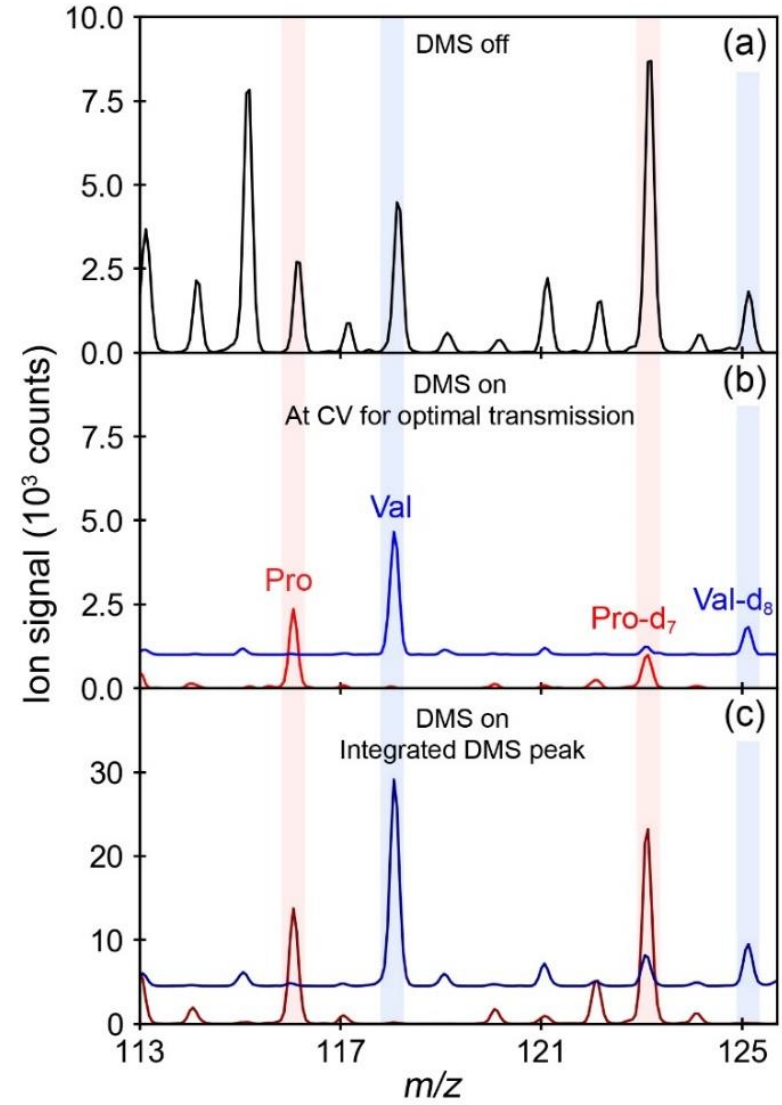

Fig. 5 Mass spectra recorded with DMS-off (black) and DMS-on (color) for a pool of plasmas sample P1. (a) DMS off and CV set at $0 \mathrm{~V}$. (b) CV set at $-5.3 \mathrm{~V}$ (red) and $-3.7 \mathrm{~V}$ (blue) for optimal transmission of protonated proline $(\mathrm{m} / \mathrm{z}$ $116)$ and valine $(\mathrm{m} / \mathrm{z} 118)$, respectively. (c) Mass spectra integrated over the DMS peaks of protonated valine (dark blue) and proline (dark red). A CV value of $1 \mathrm{~V}$ corresponds to an electric field of $1.43 \mathrm{kV} \cdot \mathrm{m}^{-1}$ or $\sim 0.07 \mathrm{Td}$.

\begin{tabular}{|l|c|c|c|c|}
\hline alanine & -7.6 & 0.3 & -7.9 & 0.3 \\
\hline serine & -6.6 & 0.2 & -6.8 & 0.2 \\
\hline proline & -5.3 & 0.1 & -5.4 & 0.2 \\
\hline valine & -3.7 & 0.1 & -3.9 & 0.2 \\
\hline threonine & -4.8 & 0.1 & -4.9 & 0.2 \\
\hline asparagine & -4.2 & 0.1 & -4.3 & 0.1 \\
\hline glutamine & -2.7 & 0.1 & -2.8 & 0.1 \\
\hline Iysine & -0.3 & $<0.1$ & -0.3 & 0.2 \\
\hline methionine & -2.3 & 0.1 & -2.4 & 0.2 \\
\hline histidine & -1.5 & 0.1 & -1.5 & 0.2 \\
\hline phenylalanine & -1.3 & 0.1 & -1.4 & 0.2 \\
\hline arginine & 0.9 & $<0.1$ & 1 & 0.2 \\
\hline citrulline & -0.7 & $<0.1$ & -0.7 & 0.2 \\
\hline tyrosine & -1.4 & 0.1 & -1.4 & $<0.1$ \\
\hline tryptophan & 0.2 & 0.1 & 0.2 & 0.1 \\
\hline
\end{tabular}

The DMS specificity is also illustrated in Figure 5. When the DMS filtering is turned on, only the targeted ions are transmitted, as well as their corresponding IS, protonated Val- $\mathrm{d}_{8}$ and Pro- $\mathrm{d}_{7}$. Note that the maximum transmission of these IS is achieved at a CV value slightly different from that of the corresponding standard. This explains the fact that the signal at $\mathrm{m} / z 126$ and 123 is lower when the DMS filtering mode is turned on than under transparent mode. The MS instrument parameters were 

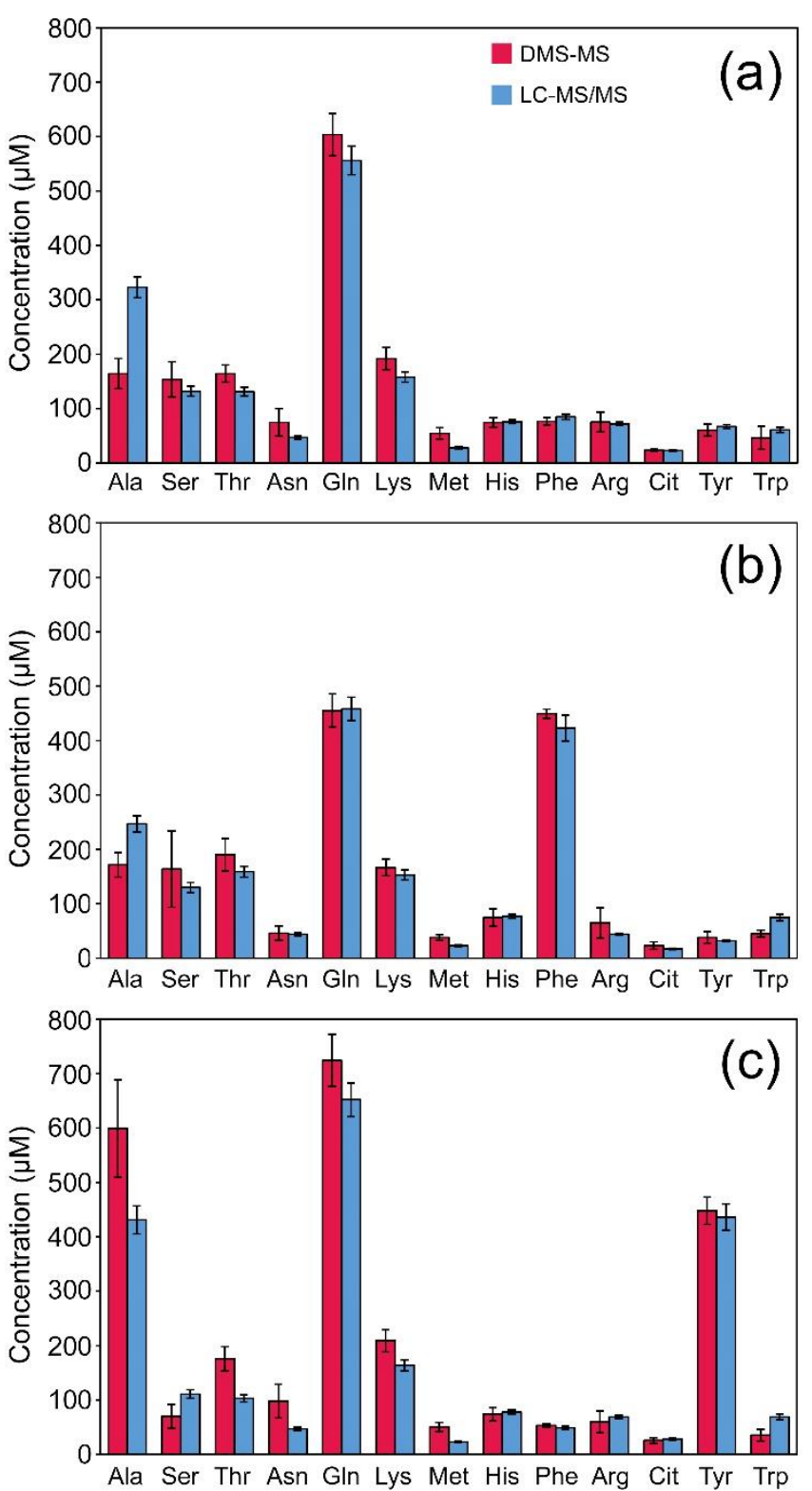

Fig. 6 Concentration $(\mu \mathrm{M})$ of 13 selected metabolites in three plasmas (P1-P3) samples. DMS-MS experiments (red bars) were performed on different days, and standard deviations are indicated as error bars. LC-MRM data are shown as blue bars, and error bars correspond to RSD calculated over 5 months.

tuned so as to avoid saturation of the trap. This is the reason why the peak intensity of protonated valine $(\mathrm{m} / z 118)$ and proline $(\mathrm{m} / \mathrm{z} 116)$ are the same using either DMS-off or DMS-on mode. If the mass spectra integrated over the DMS peaks are considered (Figure 5c), a 10-fold intensity increase is observed. A similar discussion the DMS specificity has been reported by Chen and coworkers in a study of two radiation biomarkers in human and non-human plasmas using a triple-quadrupole instrument. ${ }^{56}$

Validation of the calibration method. Calibration curves were constructed by plotting the ratio of the peak area of each reference compounds and its corresponding IS versus the corresponding reference compound concentration. Six concentration levels of the reference compounds spiked in P1 plasma were used. The results given in Table S5 were derived from 4 independent experiments performed over 6 months. In most cases, linear response profiles with $R^{2}>0.99$ were obtained in the concentration ranges of 1-300 $\mu \mathrm{M}$ after sample dilution. This concentration range covers the expected pathophysiological range for this class of molecules.

Limit of detection and limit of quantification. Limits of detection (LOD) and of quantification (LOQ) were derived from the linear regressions discussed above, where LOD can be defined as $3^{*} S D_{b} / S$, where $S$ is the slope and $S D_{b}$ is the standard deviation of the intercepts of the regression lines. This method is an alternative to the well-known signal-to-noise method when the MS analysis method does not involve background noise. ${ }^{57}$ LOD values for targeted AAs and related compounds are given in Table $\mathrm{S} 5$.

Quantification of AA levels in plasmas. Plasma samples were analyzed, and concentration levels of metabolites of interest are presented in Figure 6. Results are given for 13 AAs and related compounds. Quantification results obtained with LCMS/MS for a purpose of diagnosis of MD at the hospital are

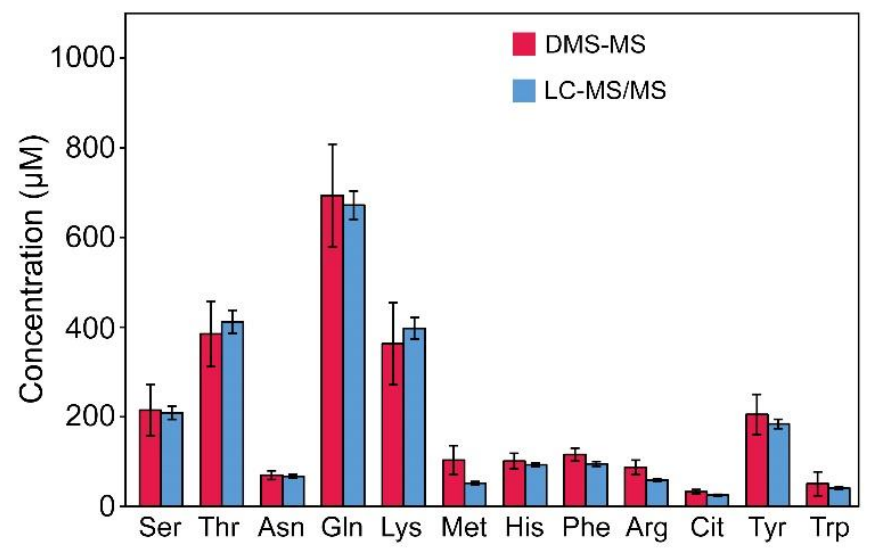

Fig. 7 Concentration $(\mu \mathrm{M})$ of selected metabolites in random plasma (P4). DMS-MS data (red bars) correspond to the mean value derived from 6 intraday experiments. Standard deviations are indicated as error bars. LC-MRM data are shown as blue bars, and error bars correspond to RSD calculated over 5 months.

given in blue bars. Results obtained with the present method are given in red bars. Inter-day quantification experiments were repeated at least three times. A calibration experiment was performed before each quantification experiment.

Concentration levels determined for plasma P1 from healthy patients are given in panel a. Overall, our method performs well compared to LC-MS/MS except in the case of Ala. This issue has been discussed above, and is probably related to the presence of $\beta$-alanine in our standard mixture used for the calibration procedure. One can also expect similar issue for the quantification of leucine, isoleucine, and allo-isoleucine (not presented). While the two formers can be separated using helium-rich carrier gas, ${ }^{22}$ these three isomers are poorly separated using $\mathrm{N}_{2}$ as a carrier gas.

Error bars for the LC-MS/MS protocol were derived for each compound from the corresponding inter-day standard deviation (SD) of an equivalent concentration calculated from plasma controls daily analyzed over a period of 5 months. For the present DMS-MS method, the error bars correspond to the 
standard deviation over the series of inter-day experiments. Taking into account the SD values, whose mean value is $18 \%$, there is a good quantitative agreement between the two approaches in $77 \%$ of the cases. As already discussed, concentration of alanine cannot be accurately determined. In the cases of lysine and methionine, concentrations are slightly overestimated using our DMS-MS approach as compared to LC$\mathrm{MS} / \mathrm{MS}$.

Inter-day quantification experiments were also carried out on two plasmas from MD patients in order to analyze AA profiles for diagnosis of known MD. Results are given for plasmas P2 and P3 (see panels b and c, respectively) from patients with Phenylketonuria and Tyrosinemia diseases, respectively. Overall, similar qualitative observations as for plasma P1 can be made. More importantly, the increase of the biomarker for these two MD diseases determined by the DMS-MS method is in excellent agreement with those obtained upon diagnosis using LC-MS/MS. In the case of Phenylketonuria P2 plasma, phenylalanine concentration was calculated at $449 \pm 8 \mu \mathrm{M}$ with our method, compared to $423 \pm 24 \mu \mathrm{M}$ with an LC-MS/MS protocol used in routine for MD diagnosis. In the case of the plasma P3, tyrosine concentration was found to be $448 \pm 25 \mu \mathrm{M}$ with DMS-MS, compared to $436 \pm 24 \mu \mathrm{M}$ with LC-MS/MS.

Intra-day quantification experiments were performed on random plasma (P4). The concentration levels determined by our method and LC-MS/MS are given in Figure 7. AAs and related metabolites profiles were analyzed 6 times, and a blank was recorded between two plasma analyses. Overall, there is a good qualitative agreement between the two sets of concentration derived by the two methods. Compared to the pool of plasmas (P1), a concentration increase of 4 out of the 13 selected metabolites (Lysine, threonine, tyrosine, methionine) is found with DMS-MS, in good agreement with LC-MS/MS. In the case of Met, however, the increase is slightly overestimated using the DMS-MS protocol. For the three other biomarkers (Lysine, threonine, tyrosine), there is a good quantitative agreement between the DMS-MS results $(363 \pm 91,385 \pm 73$, $205 \pm 45$, respectively) and the LC-MS/MS ones $(397 \pm 24,412 \pm 26$ $184 \pm 10$, respectively).

The $\mathrm{CV}$ values of the different metabolites were found stable. The mean value of the $C V$ standard deviations was $0.05 \mathrm{~V}$, lower than the overall value $(0.1 \mathrm{~V})$ derived over 6 month experiments. A similar value $(0.05 \mathrm{~V})$ was found for the mean standard deviation value of the IS. It is worth to note that the $\mathrm{CV}$ positions of standard metabolites are found to be slightly different from that of their corresponding IS. The latter were (almost) systematically found to be more positive by $0.09 \mathrm{~V}$ (mean value). Such isotopic shifts have been observed by Guevremont and coworkers for small ions such as ${ }^{37} \mathrm{Cl}^{-}$and ${ }^{35} \mathrm{Cl}^{-}{ }^{-58}$ In recent studies performed on high-definition DMS by Shvartsburg and coworkers, these isotopic CV shifts have been found to allow for identification of isomers of dichloroanilines, 59 and to be structurally informative for dibromoanilines and tribromoanilines. ${ }^{60}$

\section{Conclusions and perspectives}

We herein provide evidence that DMS hyphenated to an MS/MS instrument is a valuable alternative approach for the separation, identification, and quantification of AAs and related compounds. As retention time in LC-MS/MS, compensation voltage (CV) value allowing for the maximum transmission of a specific metabolite could allow for its identification. It is thus important to stress that the position of the DMS peak position values of metabolites of interest do not depend on concentration and/or matrix effects. Variations of $\mathrm{CV}$ positions were found to be stable within $\pm 0.1 \mathrm{~V}$, as compared to peak width (FWHM) of $0.72 \mathrm{~V}$. For a set of $\mathrm{AAs}$ and related compounds, we show here that $\mathrm{CV}$ values are reproducible over a large period of time (6 months). The proposed DMS method itself is robust since these experiments were carried out on an open MS/MS platform, and the DMS device thus had to be mounted and unmounted several times.

DMS could thus be an alternative separation method for MS/MS based targeted metabolomics for the quantification of biomarkers. In particular, two applications dedicated to MD diagnosis could be performed in order to address the topics of emergency in metabolic diagnosis and newborn rapid screening. Biomarker analysis time could be significantly reduced using DMS-MS compared to LC-MS/MS approaches. Even using an ion-trap as in the present study, a full CV scan can be performed within $\sim 5$ minutes. Furthermore, a full CV scan is no longer needed once the $\mathrm{CV}$ is known for the targeted metabolites/biomarkers. Quantification of these biomarkers could be achieved by recording mass spectra at selected identified $\mathrm{CV}$ values. Finally, as discussed in the recent literature DMS can also be hyphenated to LC-MS/MS. ${ }^{11,}$ 61-64 Two dimensional separation LC-IMS-MS/MS method allows for resolving chromatographic peaks, and an increase of signal-tonoise ratio. ${ }^{11}$

In the perspective of using DMS for identification purpose, the addition of selected organic molecules to the carrier gas is undoubtedly the approach of choice for improving DMS specificity. Drastic improvement of ion, and especially isomeric ions, separation has been observed by adding alcohol or other polar and/or proton-acceptor molecules in the buffer gas, ${ }^{23,65-}$ ${ }^{67}$ and in the case of AAs in particular. ${ }^{24,25}$ A systematic study of the effect of different modifiers on the separation of AAs and related compounds is under study in our group. Using methanol as an added modifier to $\mathrm{N}_{2}$ as carrier gas, we already showed baseline separation of isomeric ions could be obtained in the case of sarcosine, $\alpha$ - and $\beta$-alanine, ${ }^{44}$ as well as for the $\alpha-, \beta$-, and $\gamma$-aminobutyric acid isomers. ${ }^{45}$ As reported in the literature, adding of a fraction of $\mathrm{He}$ to the carrier gas $^{20,22,29}$ or increasing the waveform amplitude ${ }^{21}$ should also be considered for high resolution DMS.

Quantification of AAs and related compounds were achieved using our 3D quadrupole ion trap despite the intrinsic limitations of these instruments in terms of sensitivity and linear dynamic range. However, FT-ICR or QIT instruments are interesting for the coupling with IR lasers for DMS-peak assignment using IRMPD spectroscopy.42, 44, 45 Ultimately, however, faster quantification will be performed with DMS hyphenated to a triple quadrupole instrument. 


\section{Conflicts of interest}

There are no conflicts to declare.

\section{Acknowledgements}

Financial support from the Agence Nationale de la Recherche (ANR-19-CE29-0016-01, ANR-10-LABX-0039) is gratefully acknowledged. YW would also like to acknowledge the (CSC) for China Scholarship Council (CSC) program for a full scholarship. EA is grateful for the support of the "Programme national d'aide à I'Accueil en Urgence des Scientifiques en Exil" (PAUSE) from the Collège de France.

\section{References}

1. C. D. Chouinard, M. S. Wei, C. R. Beekman, R. H. J. Kemperman and R. A. Yost, Clin. Chem., 2016, 62, 124-133.

2. I. Kohler, A. Verhoeven, R. J. E. Derks and M. Giera, Bioanalysis, 2016, 8, 1509-1532.

3. E. Theodorsson, Bioanalysis, 2012, 4, 305-320.

4. A. Sreekumar, L. M. Poisson, T. M. Rajendiran, A. P. Khan, Q. Cao, J. D. Yu, B. Laxman, R. Mehra, R. J. Lonigro, Y. Li, M. K. Nyati, A. Ahsan, S. Kalyana-Sundaram, B. Han, X. H. Cao, J. Byun, G. S. Omenn, D. Ghosh, S. Pennathur, D. C. Alexander, A. Berger, J. R. Shuster, J. T. Wei, S. Varambally, C. Beecher and A. M. Chinnaiyan, Nature, 2009, 457, 910914.

5. D. H. Spackman, W. H. Stein and S. Moore, Anal. Chem., 1958, 30, 1190-1206.

6. M. M. Khamis, D. J. Adamko and A. El-Aneed, Mass Spectrom. Rev., 2017, 36, 115-134.

7. G. J. Patti, O. Yanes and G. Siuzdak, Nature Reviews Molecular Cell Biology, 2012, 13, 263-269.

8. H. Prinsen, B. G. M. Schiebergen-Bronkhorst, M. W. Roeleveld, J. J. M. Jans, M. G. M. de Sain-van der Velden, G. Visser, P. M. van Hasselt and N. M. Verhoeven-Duif, J. Inherit. Metab. Dis., 2016, 39, 651-660.

9. A. Desmons, E. Thioulouse, J.-Y. Hautem, A. Saintier, B. Baudin, A. Lamazière, C. Netter and F. Moussa, J. Chromatogr. A, 2020, DOI: j.chroma.2020.461135, 461135 H. S. Jonasdottir, C. Papan, S. Fabritz, L. Balas, T. Durand, I. Hardardottir, J. Freysdottir and M. Giera, Anal. Chem., 2015, 87, 5036-5040.

11. P. R. S. Baker, A. M. Armando, J. L. Campbell, O. Quehenberger and E. A. Dennis, J. Lipid Res., 2014, 55, 2432-2442.

12. T. P. I. Lintonen, P. R. S. Baker, M. Suoniemi, B. K. Ubhi, K. M. Koistinen, E. Duchoslav, J. L. Campbell and K. Ekroos, Anal. Chem., 2014, 86, 9662-9669.

13. A. Basit, S. Pontis, D. Piomelli and A. Armirotti, Metabolomics, 2016, 12, 10.

14. C. Lapthorn, F. Pullen and B. Z. Chowdhry, Mass Spectrom. Rev., 2013, 32, 43-71.

$15 . \quad$ B. B. Schneider, E. G. Nazarov, F. Londry, P. Vouros and T. R. Covey, Mass Spectrom. Rev., 2016, 35, 687-737.

16. P. Dwivedi, A. J. Schultz and H. H. Hill, Int. J. Mass spectrom., 2010, 298, 78-90.
17. G. Paglia and G. Astarita, Nature Protocols, 2017, 12, 797 813.

18. F. A. Fernandez-Lima, D. A. Kaplan and M. A. Park, Rev. Sci. Instrum., 2011, 82.

19. I. K. Webb, S. V. B. Garimella, A. V. Tolmachev, T. C. Chen, X. Y. Zhang, R. V. Norheim, S. A. Prost, B. LaMarche, G. A. Anderson, Y. M. Ibrahim and R. D. Smith, Anal. Chem., 2014, 86, 9169-9176.

20. M. A. Baird, P. V. Shliaha, G. A. Anderson, E. Moskovets, V. Laiko, A. A. Makarov, O. N. Jensen and A. A. Shvartsburg, Anal. Chem., 2019, 91, 6918-6925.

21. M. A. Baird, G. A. Anderson, P. V. Shliaha, O. N. Jensen and A. A. Shvartsburg, Anal. Chem., 2019, 91, 1479-1485.

22. A. A. Shvartsburg, W. F. Danielson and R. D. Smith, Anal. Chem., 2010, 82, 2456-2462.

23. R. Fernandez-Maestre, C. Wu and H. H. Hill, Rapid Commun. Mass Spectrom., 2012, 26, 2211-2223.

24. D. G. Beach, E. S. Kerrin, S. D. Giddings, M. A. Quilliam and P. McCarron, Sci Rep, 2018, 8, 11

25. M. McCooeye and Z. Mester, Rapid Commun. Mass Spectrom., 2006, 20, 1801-1808.

26. R. Perez-Miguez, B. Bruyneel, M. Castro-Puyana, M. L. Marina, G. W. Somsen and E. Dominguez-Vega, Anal. Chem., 2019, 91, 3277-3285.

27. J. D. Zhang, K. M. M. Kabir, H. E. Lee and W. A. Donald, Int. J. Mass spectrom., 2018, 428, 1-7.

28. K. J. D. Fouque, A. Garabedian, J. Porter, M. Baird, X. Q Pang, T. D. Williams, L. J. Li, A. Shvartsburg and F. Fernandez-Lima, Anal. Chem., 2017, 89, 11787-11794.

29 D. L. Winter, J. Mastellone, K. M. M. Kabir, M. R. Wilkins and W. A. Donald, Anal. Chem., 2019, 91, 11827-11833.

30. M. Hernandez-Mesa, A. Escourrou, F. Monteau, B. Le Bizec and G. Dervilly-Pinel, Trac-Trends in Analytical Chemistry, 2017, 94, 39-53.

31. R. Guevremont, D. A. Barnett, R. W. Purves and J. Vandermey, Anal. Chem., 2000, 72, 4577-4584.

32. S. Wernisch, F. Afshinnia, T. Rajendiran and S. Pennathur, Analytical and Bioanalytical Chemistry, 2018, 410, 28652877.

33. E. Sinclair, K. A. Hollywood, C. Y. Yan, R. Blankley, R. Breitling and P. Barran, Analyst, 2018, 143, 4783-4788.

S. Goscinny, L. Joly, E. De Pauw, V. Hanot and G. Eppe, J. Chromatogr. A, 2015, 1405, 85-93.

35. J. Regueiro, N. Negreira, R. Hannisdal and M. H. G. Berntssen, Food Control, 2017, 78, 116-125.

36. J. Regueiro, N. Negreira and M. H. G. Berntssen, Anal. Chem., 2016, 88, 11169-11177.

37. Z. W. Zhou, X. T. Shen, J. Tu and Z. J. Zhu, Anal. Chem., 2016, 88, 11084-11091.

38. X. Y. Zheng, N. A. Aly, Y. X. Zhou, K. T. Dupuis, A. Bilbao, V. L. Paurus, D. J. Orton, R. Wilson, S. H. Payne, R. D. Smith and E. S. Baker, Chem. Sci., 2017, 8, 7724-7736.

39. C. M. Nichols, J. N. Dodds, B. S. Rose, J. A. Picache, C. B. Morris, S. G. Codreanu, J. C. May, S. D. Sherrod and J. A. McLean, Anal. Chem., 2018, 90, 14484-14492.

40. L. C. Nye, J. P. Williams, N. C. Munjoma, M. P. M. Letertre, M. Coen, R. Bouwmeester, L. Martens, J. R. Swann, J. K. Nicholson, R. S. Plumb, M. McCullagh, L. A. Gethings, S. Lai, J. I. Langridge, J. P. C. Vissers and I. D. Wilson, J. Chromatogr. A, 2019, 1602, 386-396. 
41. L. D. Roberts, A. L. Souza, R. E. Gerszten and C. B. Clish, current protocols in molecular biology, 2012, 98, 30.32.3130.32.24.

42. O. Hernandez, S. Isenberg, V. Steinmetz, G. L. Glish and P. Maitre, J. Phys. Chem. A, 2015, 119, 6057-6064.

43. S. L. Isenberg, P. M. Armistead and G. L. Glish, J. Am. Soc. Mass. Spectrom., 2014, 25, 1592-1599.

44. F. Berthias, B. Maatoug, G. Glish, F. Moussa and P. Maitre, J. Am. Soc. Mass. Spectrom., 2018, 29, 752-760.

45. Y. Wang, E. Alhajji, B. Rieul, F. Berthias and P. Maitre, Int. J. Mass Spectrom. Ion Processes, 2019, 443, 16-21.

46. A. A. Shvartsburg, Differential Mobility Spectrometry: Nonlinear lon Transport and Fundamentals of FAIMS CRC Press, 2008.

47. I. A. Buryakov, E. V. Krylov, E. G. Nazarov and U. K. Rasulev, Int. J. Mass Spectrom. Ion Processes, 1993, 128, 143-148.

48. J. L. Campbell, M. Zhu and W. S. Hopkins, J. Am. Soc. Mass. Spectrom., 2014, 25, 1583-1591.

49. A. A. Shvartsburg, K. Q. Tang, R. D. Smith, M. Holden, M. Rush, A. Thompson and D. Toutoungi, Anal. Chem., 2009, 81, 8048-8053.

50. E. V. Krylov, S. L. Coy, J. Vandermey, B. B. Schneider, T. R. Covey and E. G. Nazarov, Rev. Sci. Instrum., 2010, 81.

51. K. L. Davidson and M. F. Bush, Anal. Chem., 2017, 89, 20172023.

52. L. W. Beegle, I. Kanik, L. Matz and H. H. Hill, Anal. Chem., 2001, 73, 3028-3034.

53. G. R. Asbury and H. H. Hill, J. Chromatogr. A, 2000, 902, 433-437.

54. M. R. Menlyadiev, A. Tarassov, A. M. Kielnecker and G. A. Eiceman, Analyst, 2015, 140, 2995-3002.

55. M. T. Campbell and G. L. Glish, Int. J. Mass spectrom., 2018, 425, 47-54.

56. Z. D. Chen, S. L. Coy, E. L. Pannkuk, E. C. Laiakis, A. B. Hall, A. J. Fornace and P. Vouros, J. Am. Soc. Mass. Spectrom., 2016, 27, 1626-1636.

57. M. M. Sanagi, S. L. Ling, Z. Nasir, D. Hermawan, W. A. W. Ibrahim and A. Abu Naim, J. AOAC Int., 2009, 92, 18331838.

58. D. A. Barnett, R. W. Purves and R. Guevremont, Nuclear Instruments \& Methods in Physics Research Section aAccelerators Spectrometers Detectors and Associated Equipment, 2000, 450, 179-185.

59. P. Pathak, M. A. Baird and A. A. Shvartsburg, Anal. Chem., 2018, 90, 9410-9417.

60. P. Pathak, M. A. Baird and A. A. Shvartsburg, J. Am. Soc. Mass. Spectrom., 2020, 31, 137-145.

61. D. G. Beach, J. Am. Soc. Mass. Spectrom., 2017, 28, 15181530.

62. E. Varesio, J. C. Y. Le Blanc and G. Hopfgartner, Analytical and Bioanalytical Chemistry, 2012, 402, 2555-2564.

63. H. B. Xu, F. R. Boucher, T. T. Nguyen, G. P. Taylor, J. J. Tomlinson, R. A. Ortega, B. Simons, M. G. Schlossmacher, R. Saunders-Pullman, W. Shaw and S. A. L. Bennett, J. Lipid Res., 2019, 60, 200-211.

64. Y. L. Fu, Y. Q. Xia, J. Flarakos, F. L. S. Tse, J. D. Miller, E. B. Jones and W. K. Li, Anal. Chem., 2016, 88, 3655-3661.

65. G. A. Eiceman, E. V. Krylov, E. G. Nazarov and R. A. Miller, Anal. Chem., 2004, 76, 4937-4944.

66. N. Krylova, E. Krylov, G. A. Eiceman and J. A. Stone, J. Phys. Chem. A, 2003, 107, 3648-3654.
67. D. Ruskic and G. Hopfgartner, Anal. Chem., 2019, 91, 11670-11677. 